\title{
Las variaciones del tipo de cambio y el índice de inflación en las economías emergentes
}

\author{
José García-Solanes y Fernando Torrejón-Flores
}

RESUMEN

Se propone un modelo de equilibrio general estructural para analizar las reacciones del tipo de cambio nominal y el nivel de precios internos ante tres tipos de shocks externos en economías emergentes con acceso limitado a los mercados mundiales de capital. Aunque los resultados dependen decisivamente del tipo de shock externo, los parámetros del balance nacional aquí examinados, la prima de riesgo y el índice de endeudamiento externo exacerban las reacciones de las dos variables endógenas, sin alterar el grado de traspaso del tipo de cambio. Además, las curvas de Phillips más planas, como las observadas actualmente en muchas economías, tienden a aumentar el traspaso del tipo de cambio. A partir de estos resultados se recomienda a las autoridades de las economías emergentes, que desean estabilizar los mercados y limitar el traspaso del tipo de cambio, minimizar los dos parámetros de riesgo mediante un régimen de metas de inflación flexible.

PALABRAS CLAVE

CLASIFICACIÓN JEL

AUTORES
Tipos de cambio, precios, mercados de capital, modelos econométricos, macroeconomía, mercados emergentes

E52, F21, F33

José García-Solanes es catedrático de Análisis Económico de la Universidad de Murcia, España. solanes@um.es Fernando Torrejón-Flores es profesor de Análisis Económico de la Universidad Católica de San Antonio de Murcia, España. ftorrejon@ucam.edu 


\section{I}

\section{Introducción}

La literatura sobre las variaciones en los tipos de cambio y los precios en las economías abiertas se ha concentrado tradicionalmente en el traspaso del tipo de cambio (exchange rate pass trhough), es decir, la medida en que las fluctuaciones cambiarias se transmiten a los precios internos. Para evaluar los efectos puros del traspaso del tipo de cambio, es necesario limitar el análisis a las repercusiones iniciales en los precios de importación o, a lo sumo, a las primeras etapas que afectan a los precios internos al productor. $\mathrm{Al}$ extender el análisis a etapas posteriores, que incluyen el equilibrio de los mercados de bienes y activos, el cuociente final entre las variaciones de los precios internos y las fluctuaciones de la tasa de cambio ofrece una medida más indirecta del traspaso del tipo de cambio. Como destaca Mishkin (2008), las relaciones entre los tipos de cambio y los precios internos son sensibles a la naturaleza de los shocks externos que desencadenan los ajustes.

Si bien en general se considera que los bajos índices de inflación, la credibilidad de la política monetaria y la flexibilidad del tipo de cambio contribuyen a disminuir el traspaso del tipo de cambio, algunos autores realizaron estimaciones que contradicen o ponen en tela de juicio ese consenso ${ }^{1}$. Por ejemplo, Nogueira (2007) observó que la adopción de un régimen de metas de inflación -que refuerza la credibilidad de la política monetariaincrementó el traspaso del tipo de cambio en la República Checa y la República de Corea, mientras que Coricelli, Jazbec y Masten (2004) descubrieron que la flexibilidad cambiaria aumentó el coeficiente de traspaso del tipo de cambio en cuatro economías en transición avanzadas. En una línea similar, Byrne, Chavali y Kontonikas (2010);

$\square$ Los autores agradecen el apoyo financiero de la Fundación Séneca (proyecto 15183/PHCS/10).

1 Taylor (2000); Korhonen y Watchel (2006); Choundri y Hakura (2006), y Mihaljek y Klau (2008) observaron que el traspaso del tipo de cambio se reduce en los contextos de baja inflación. Mishkin y Savastano (2002); Vega y Winkelried (2005); Nogueira (2007), y Nogueira y León-Ledesma (2011), entre otros, subrayaron la capacidad de la credibilidad de la política monetaria de disminuir el traspaso del tipo de cambio. Por último, Barhoumi (2006); Sek y Kapsalyamova (2008), y Takatoshi y Kiyotaka (2008) encontraron que la flexibilidad cambiaria disminuye el traspaso del tipo de cambio al incrementar la volatilidad en el mercado cambiario y reducir el comercio.
Sek y Kapsalyamova (2008), y Korhonen y Wachtel (2006) hallaron que los efectos de dicho traspaso son, de hecho, muy heterogéneos y asimétricos entre los países. De acuerdo con Mishkin (2008), las divergencias en esos resultados pueden explicarse porque los autores no tuvieron en cuenta el tipo de shocks externos que afectaron a las economías.

En este trabajo se examina el traspaso del tipo de cambio, que se obtiene cuando los tipos de cambio y los precios internos se ajustan en el macroequilibrio a corto plazo. Para abordar el problema planteado por Mishkin (2008) se analizan por separado los efectos de tres shocks externos distintos. Se examina, además, la manera en que cuatro características de las economías emergentes inciden en los ajustes: el grado de rigidez del tipo de cambio, el nivel de riesgo país, el endeudamiento de este y el aplanamiento de las curvas de Phillips desde el comienzo de la Gran Recesión (2007-2012)².

Para ello, se construye un modelo de equilibrio general estructural centrado en la flexibilidad del tipo de cambio y los efectos de balance. En comparación con modelos anteriores para las economías emergentes, en este se incorporan dos importantes novedades: en primer lugar, desde el punto de vista de la producción, se deriva una función de oferta agregada que incluye la inercia en la fijación de precios, conforme con la nueva curva de Phillips keynesiana para las economías abiertas y, en segundo lugar, se genera una función de política monetaria óptima, al asumir que los bancos centrales procuran minimizar las pérdidas intertemporales ocasionadas por las brechas de producción y las desviaciones de la inflación y del tipo de cambio nominal con respecto a sus respectivas metas.

Las desviaciones de las variables endógenas de su nivel estacionario se calculan como funciones de los parámetros exógenos, y se realizan ejercicios de calibración después de asignar a los parámetros del modelo valores generalmente aceptados en la

\footnotetext{
2 En FMI (2013, cap. 3) se ofrece una descripción detallada de los factores que probablemente han disminuido la volatilidad de la inflación y su sensibilidad a los cambios en la brecha de producción con respecto al pasado, incluidos los "costos de menú".
} 
literatura. Este procedimiento permite establecer la manera en que los efectos de los shocks exógenos se transmiten a través de cuatro canales antes de producir un impacto en el tipo de cambio y el índice de precios al productor interno. Debido al particular interés de los autores del presente estudio en averiguar las repercusiones de la rigidez del tipo de cambio, el riesgo país, el endeudamiento de este y la pendiente de la curva de Phillips en este proceso, se realizan calibraciones para distintos valores de estos parámetros.

Se destacan dos resultados clave. En primer lugar, la rigidez del tipo de cambio, la estabilidad macroeconómica - en la medida en que reduce los parámetros de riesgo y endeudamiento- - y las curvas de Phillips más planas disminuyen las fluctuaciones cambiarias y de los precios internos cuando los shocks consisten en variaciones de los precios y de la producción en el exterior. Sin embargo, la rigidez del tipo de cambio y las curvas de Phillips más planas intensifican las fluctuaciones de las dos variables endógenas cuando los ajustes obedecen a variaciones en la tasa de interés real externa. Solo la estabilidad macroeconómica puede mitigar las fluctuaciones en las dos variables endógenas causadas por cualquier tipo de

\section{II}

\section{Modelo teórico}

En esta sección se construye y resuelve un modelo de equilibrio general estructural para analizar los efectos de varios shocks externos en el tipo de cambio nominal y en los precios internos de una economía emergente pequeña y abierta que enfrenta imperfecciones en los mercados financieros internacionales. Se amplía el trabajo de Céspedes, Chang y Velasco (2003 y 2004); Fraga, Goldfajn y Minella (2003), y Tovar (2005).

Las economías comprenden cinco tipos de agentes: empresas, hogares, capitalistas, gobiernos y autoridades monetarias. Hay un gran número de empresas que producen bienes diferenciados en un contexto de competencia monopolística. Las empresas obtienen capital de los capitalistas y mano de obra de los hogares. Los capitalistas deciden el tamaño de sus inversiones, que financian parcialmente con sus propios recursos (valor neto) y en parte con deuda externa denominada shock externo. En segundo lugar, después de cada shock externo examinado en el análisis, el traspaso del tipo de cambio aumenta con la mayor rigidez cambiaria y con las curvas de Phillips más planas, pero resulta apenas afectado por las variaciones en los factores de riesgo y endeudamiento. La principal consecuencia es que la reducción de los parámetros de estos factores acrecienta la capacidad de la política monetaria de estabilizar tanto las variaciones del tipo de cambio, como la inflación interna cuando la economía padece cualquiera de los tres shocks examinados en el análisis, sin afectar al grado de traspaso del tipo de cambio.

El presente trabajo se divide en cuatro secciones incluida esta Introducción. Mientras que en la segunda sección se presenta y se resuelve el modelo teórico para las variables endógenas seleccionadas, en la tercera sección se calibra el modelo para evaluar los efectos de los cambios de algunas variables exógenas en el tipo de cambio nominal y en el índice de precios internos, y se calculan los coeficientes de traspaso del tipo de cambio provocados por cada shock. Para concluir, en la cuarta sección se examinan algunas repercusiones de los resultados desde el punto de vista normativo. en moneda extranjera fuerte. Los hogares nacionales emiten bonos expresados en una moneda extranjera fuerte y optimizan sus acciones teniendo en cuenta las restricciones presupuestarias intertemporales del gobierno. Los gobiernos recaudan impuestos globales para financiar sus gastos de consumo. Las autoridades monetarias se ocupan de la estabilización de tres variables: producción nacional, índice de inflación interno y tipo de cambio nominal. Esto supone que, para responder a los shocks externos, los bancos centrales toman medidas destinadas a lograr la combinación óptima de las tres variables.

A corto plazo, se supone que los precios se ajustan lentamente, de acuerdo con Calvo (1983). El número de empresas que cambian los precios en un período determinado se especifica de manera exógena en este marco. Las variables del modelo se presentan como desviaciones lineales respecto de su estado estacionario, 
con excepción de las tasas de interés y las primas de riesgo $^{3}$. El modelo está compuesto por las siguientes ecuaciones ${ }^{4}$ :

$$
\begin{gathered}
\frac{1}{2} E_{t}\left\{\sum _ { i = 0 } ^ { \infty } \beta ^ { i } \left[w_{\tilde{y}}\left(\tilde{y}_{t+i}\right)^{2}+w_{s}\left(s_{t+i}-s_{t+i}^{T}\right)^{2}\right.\right. \\
\left.\left.+w_{\pi}\left(\pi_{t+i}^{H}-\pi_{t+i}^{H T}\right)^{2}\right]\right\} \\
\pi_{t+i}^{H}=\lambda_{r} r e_{t+i}+\lambda_{\tilde{y}} \tilde{y}_{t+i}+\lambda_{\tilde{y}} \tilde{y}_{t+i}^{*}+\lambda_{q} q_{t+i} \\
+\beta E_{t}\left(\pi_{t+1+i}^{H}\right)+\mu_{t+i} \\
y_{t}=s_{c} h_{y} E_{t}\left(y_{t+1}\right)+s_{c} h_{q} E_{t}\left(q_{t+1}\right)+s_{c} h_{b^{*}} E_{t}\left(b_{t+1}^{*}\right) \\
-s_{c} h_{b^{*}} b_{t}^{*}-\frac{s_{c}}{\gamma_{c}} r_{t}+\left(s_{q}+s_{x} \eta\right) q_{t}+s_{x} y_{t}^{*}+s_{g} g_{t} \\
-s_{c} h_{g} E_{t}\left(g_{t+1}\right)+s_{c} \phi_{t}+s_{i n} i n_{t} \\
r_{t}-r_{t}^{*}=\psi\left[E_{t}\left(q_{t+1}\right)-q_{t}\right]+\zeta_{t} \\
\zeta_{t}=\iota[(1-\gamma)+\chi] q_{t}+\iota i n_{t}-\iota(1+\chi) y_{t}+\iota \chi d e_{t}+\vartheta_{t} \\
i n_{t}=E_{t}\left(y_{t+1}\right)-[1+(1-\gamma)(1-\delta)] E_{t}\left(q_{t+1}\right) \\
+[1-(1-\gamma) \delta] q_{t}-r_{t}^{*}-\zeta_{t}^{*}
\end{gathered}
$$

La ecuación (1) es una función de pérdida intertemporal del banco central que penaliza las desviaciones de la producción, del tipo de cambio nominal y del índice de inflación con respecto a sus metas. El logaritmo de la brecha de producción $\tilde{y}_{t}$ se calcula con relación al nivel de producción a largo plazo o potencial $\left(\bar{y}_{t}\right) \cdot s_{t}^{T}$ es el logaritmo de la meta del tipo de cambio nominal anunciada por el banco central, que se define como el precio de la moneda extranjera en unidades de la moneda nacional. $\pi_{t}^{T}$ es la meta del índice de inflación. La inclusión de desviaciones del

\footnotetext{
${ }^{3}$ Las desviaciones relativas con respecto al estado estacionario se denotan con una letra minúscula. Por ejemplo, en el caso de la variable $X_{t}$, cuyo nivel estacionario es $X^{S S}$, la desviación relativa se define como: $x_{t}=\left(X_{t}-X^{S S}\right) / X^{S S}$.

La desviación relativa se puede expresar como un valor muy aproximado mediante: $x_{t}=\ln \left(X_{t}-X^{S S}\right)$.

${ }^{4}$ Estas ecuaciones se han derivado suponiendo que todos los agentes optimizan su comportamiento. Es posible solicitar una explicación más detallada a los autores.
}

tipo de cambio con respecto a la meta en la función de pérdida del banco central se justifica por dos razones: en primer lugar, es probable que en las economías abiertas examinadas las fluctuaciones del tipo de cambio afecten a la oferta y la demanda agregada en forma significativa, y en segundo lugar, la volatilidad del tipo de cambio nominal modifica el patrimonio neto de los capitalistas, porque su endeudamiento externo está denominado en monedas extranjeras fuertes.

Si bien algunos autores han reconocido la importancia de la política del tipo de cambio al incluirla en la función de reacción monetaria (por ejemplo: Ball, 1999; Obstfeld y Rogoff, 1995; Svensson, 2000; Mishkin y Savastano, 2002; Caballero y Krishnamurthy, 2005, y Wollmershäuser, 2003), el modelo construido en este trabajo es el primero que incorpora el tipo de cambio en la función de pérdida del banco central. De acuerdo con Edwards (2006), aunque los bancos centrales no lo reconocen en forma explícita, son muchos los casos en que el tipo de cambio desempeña un papel importante en la función de reacción de política monetaria (reglas de Taylor).

$E_{t}$ es el operador de expectativas racionales en el período $t, \beta$ es el factor de descuento y $W_{y}$ representa el peso relativo asignado a la variabilidad de la producción. La especificación de la función de pérdida en el presente estudio permite, como en Tovar (2005), prever un continuo de regímenes cambiarios según el peso relativo asignado a la estabilización del tipo de cambio ${ }^{5}$. Los parámetros $W_{s}$ y $W_{\pi}$ miden el peso relativo asignado, respectivamente, a la variabilidad cambiaria y al índice de inflación.

La ecuación (2) es una oferta agregada de acuerdo con el espíritu de la nueva curva de Phillips keynesiana que incorpora la inercia en la fijación de precios. En la versión aquí presentada se incluyen dos variables de economía abierta: el logaritmo del tipo de cambio real, que transmite las perturbaciones externas - como el aumento de los precios externos-a la inflación interna, y el logaritmo de la brecha de producción externa $\left(\tilde{y}_{t}^{*}\right)$. El tipo de cambio real se define de manera que un aumento denote la depreciación real de la moneda nacional. La tasa de rendimiento del capital $\left(r e_{t}\right)$ se incorpora a la ecuación como un factor adicional de incremento de los costos. La parte convencional de la ecuación se puede derivar asumiendo — al igual que en Calvo (1983) — que las empresas maximizan la diferencia entre sus ingresos marginales esperados y su costo unitario, y que solo una

\footnotetext{
5 En el modelo de Tovar, la diversidad de los regímenes de tipo de cambio obedece a las diferencias en el peso asignado a la estabilización cambiaria según la regla de Taylor.
} 
parte de ellas ajusta los precios durante cada período. Todos los coeficientes $\lambda_{r e}, \lambda_{\tilde{y}}, \lambda_{\tilde{y}} * \mathrm{y} \lambda_{q}$ son positivos y proporcionales al parámetro $\lambda$, que se vincula con la probabilidad de ajustar los precios en el período actual $(1-\theta)$ con esta expresión: $(\lambda=(1-\theta)(1-\theta \beta) / \theta)$. De ese modo, la oferta agregada, así como la curva de Phillips implícita, se aplanan ( $\lambda_{\tilde{y}}$ disminuye $)$ a medida que se reduce la probabilidad de ajustar los precios ${ }^{6}$. La variable $\mu_{t}$ es una perturbación exógena de oferta que acrecienta la inflación.

La ecuación (3) indica que la demanda agregada depende positivamente del tipo de cambio real (mecanismo de reorientación de gastos), la producción esperada (debido a la disminución del consumo de los hogares) ${ }^{7}$, el tipo de cambio real esperado, la variación esperada de la deuda externa denominada en moneda extranjera $\left(b_{t}^{*}\right)$, la producción externa $\left(y_{t}^{*}\right)$, los gastos del gobierno $\left(g_{t}\right)$, la inversión interna $\left(i n_{t}\right)$ y una perturbación de la demanda $\left(\phi_{t}\right)$. La demanda agregada se aminora a medida que aumentan la tasa de interés real $\left(r_{t}\right)$ y los gastos esperados del gobierno.

La ecuación (4) es la condición de paridad descubierta de las tasas de interés expresada en términos reales. La variable $\xi_{t}$ es la prima de riesgo país, que se determina de forma endógena de acuerdo con la ecuación (5). La última ecuación indica que la prima de riesgo aumenta de manera inequívoca cuando el valor de la inversión corriente crece y el tipo de cambio real se deteriora - la depreciación real incrementa el valor de los pagos de la deuda en caso de dolarización del pasivo. También se acrecienta con la proporción de la deuda de los capitalistas denominada en moneda extranjera $\left(d e_{t}\right)$ y en caso de shock estocástico $\left(\vartheta_{t}\right)$. La prima de riesgo disminuye cuando aumenta la producción nacional, pues esta última se relaciona con los ingresos y el valor neto de los capitalistas. Como puede observarse, la incidencia de estos factores determinantes de la prima de riesgo depende en forma decisiva del valor de los parámetros ı y $\chi$, que son —respectivamente— la semielasticidad de la prima de riesgo ligada, respectivamente, a la relación entre la inversión y el patrimonio neto de los capitalistas y a la relación entre la deuda externa y el patrimonio neto.

Por último, la ecuación (6) corresponde a la demanda de inversión, que puede derivarse fácilmente del arbitraje

\footnotetext{
${ }^{6}$ Cuando la probabilidad de ajustar los precios equivale a uno, lo que significa que todas las empresas pueden cambiar sus precios en cualquier momento (flexibilidad total de los precios), la oferta agregada está representada por una línea totalmente vertical (véase, por ejemplo, Woodford (2003, capítulo 2)).

7 Véase, por ejemplo, Fraga, Goldfajn y Minella (2003).
}

internacional estándar sobre las tasas de rentabilidad. Esta relación establece que la inversión interna disminuye al aumentar el costo internacional del capital (la suma de la tasa de interés mundial y la prima de riesgo), debido a que los capitalistas se endeudan en el exterior para financiar la inversión. La inversión interna también se reduce con el tipo de cambio real esperado porque, manteniéndose constantes las demás condiciones, un $q_{t}$ esperado más alto hoy se asocia con una depreciación real esperada más alta entre hoy y mañana, y por ende con un costo mayor del capital extranjero, que se mide en términos de bienes nacionales. Asimismo, la inversión interna depende positivamente de la producción esperada y del tipo de cambio real corriente porque, manteniéndose constantes las demás condiciones, un $q_{t}$ más alto hoy se asocia con una depreciación real esperada inferior. Los parámetros $\gamma$ y $\delta$ representan la preferencia por el consumo de bienes nacionales (sesgo nacional) y la proporción de los flujos internacionales del país de origen en el comercio internacional mundial.

$\mathrm{Al}$ combinar las ecuaciones (5) y (6) se obtiene la curva de la balanza de pagos (BP), el lugar geométrico de los puntos $\left(y_{t}, i n_{t}\right)$ en que los mercados financieros están en equilibrio, todo lo demás constante:

$$
\begin{gathered}
i n_{t}=\frac{1}{1+\iota} E_{t}\left(y_{t+1}\right)+\frac{\iota(1+\chi)}{1+\iota} y_{t} \\
-\frac{1+(1-\gamma)(1-\delta)}{1+\iota} E_{t}\left(q_{t+1}\right) \\
+\frac{[1-(1-\gamma) \delta]-\iota[(1-\gamma)+\chi]}{1+\iota} q_{t} \\
-\frac{\iota \chi}{1+\iota} d e_{t}-\frac{1}{1+\iota} r_{t}^{*}-\frac{1}{1+\iota} \vartheta_{t}
\end{gathered}
$$

La curva BP ilustra la manera en que el grado de imperfección de los mercados de capital $(\mathrm{\iota})$ y la posición financiera internacional del país $(\chi)$ afectan a la inversión. En particular, como se muestra en Céspedes, Chang y Velasco (2003), la inversión puede aumentar o disminuir con el tipo de cambio real. Cuando las imperfecciones del mercado de capital y la deuda en dólares heredada son lo suficientemente altas (valores elevados de $\iota \mathrm{y}$ $\chi$ ), el efecto de balance predomina sobre el efecto de reorientación de gastos relacionado con el coeficiente $\gamma$. En ese caso, el coeficiente de $q_{t}$ es negativo y la economía se vuelve vulnerable desde el punto de vista financiero. En comparación con el marco de Céspedes, Chang y Velasco (2003), el coeficiente de $q_{t}$ del modelo utilizado en este trabajo refuerza la influencia del canal 
de reorientación de gastos, porque se incluye el parámetro $\delta<1$, que es igual a la unidad en el modelo anterior. Un caso particular interesante, también destacado por esos autores, es el de la ausencia de imperfecciones financieras $(l=0)$, que da lugar a una BP horizontal en el espacio $\left(y_{t}, i n_{t}\right)$.

La solución del modelo para las dos variables endógenas seleccionadas en el presente estudio - el nivel de precios al productor $\left(p_{t}^{H E}\right)$ y el tipo de cambio nominal $\left(s_{t}^{E}\right)$ - se presenta en el anexo. El modelo tiene 14 variables exógenas: producción externa $\left(y_{t}^{*}\right)$; producción externa potencial $\left(\bar{y}_{t}^{*}\right)$; crisis de la oferta que incrementa los costos $\left(\mu_{t}\right)$; productividad media de los factores de producción (estado de la tecnología) $\left(a_{t}\right)$; bonos denominados en moneda extranjera emitidos por residentes nacionales, en manos de extranjeros (deuda externa) $\left(b_{t}^{*}\right)$; gastos del gobierno $\left(g_{t}\right)$; crisis de la demanda en el consumo privado $\left(\phi_{t}\right)$; tasa de interés real externa $\left(r_{t}^{*}\right)$; tasa de rendimiento del capital $\left(r e_{t}\right)$; nivel de precios externos $\left(p_{t}^{*}\right)$; deuda externa de los capitalistas $\left(d e_{t}\right)$; meta de tipo de cambio nominal $\left(s_{t}^{T}\right)$; meta de índice de inflación interna $\left(\pi_{t}^{H T}\right)$, y shock que afecta a la prima de riesgo $\left(\vartheta_{t}\right)$. Asimismo, todas las variables exógenas — con excepción de $s_{t}^{T}$ y $\pi_{t}^{H T}$ están gobernadas por procesos estacionarios $\operatorname{AR}(1)$ :

$$
\begin{gathered}
y_{t}^{*}=\rho_{y^{*}} y_{t-1}^{*}+\varepsilon_{y^{*}, t}, \bar{y}_{t}^{*}=\rho_{\bar{y}^{*}} \bar{y}_{t-1}^{*}+\varepsilon_{\bar{y}_{t}^{*}, t}, \\
\mu_{t}=\rho_{\mu} \mu_{t-1}+\varepsilon_{\mu, t}, a_{t}=\rho_{a} a_{t-1}+\varepsilon_{a, t}, \\
b_{t}^{*}=\rho_{b^{*}} b_{t-1}^{*}+\varepsilon_{b^{*}, t}, g_{t}=\rho_{g} g_{t-1}+\varepsilon_{g, t}, \\
\phi_{t}=\rho_{\phi} \phi_{t-1}+\varepsilon_{\phi, t}, r_{t}^{*}=\rho_{r^{*}} r_{t-1}^{*}+\varepsilon_{r^{*}, t}, \\
r e_{t}=\rho_{r} r e_{t-1}+\varepsilon_{r e, t}, p_{t}^{*}=\rho_{p^{*}} p_{t-1}^{*}+\varepsilon_{p^{*}, t}, \\
d e_{t}=\rho_{d e} d e_{t-1}+\varepsilon_{d e, t}, \vartheta_{t}=\rho_{\vartheta} \vartheta_{t-1}+\varepsilon_{\vartheta, t}
\end{gathered}
$$

Las ecuaciones de equilibrio del nivel de precios interno y del tipo de cambio nominal, incluidos los signos de las derivadas parciales con respecto a los parámetros exógenos, se presentan en el anexo (ecuaciones (A7) y (A8)).

Antes de calibrar el modelo, se discute brevemente el aporte relativo de cada canal en la transmisión de los efectos después de la depreciación inicial del tipo de cambio nominal ${ }^{8}$. El canal de gastos produce efectos

\footnotetext{
8 En general, se acepta que los shocks externos afectan en primer lugar a los mercados de activos, incluido el mercado de divisas. El
}

expansivos en la demanda agregada y en el modelo aquí utilizado está compuesto por dos elementos. El primero refleja el efecto del traspaso inicial: el aumento del tipo de cambio nominal provoca el alza del nivel de precios agregados, que - a su vez-incrementa el valor nominal de los gastos internos. El segundo es la reorientación de gastos: la depreciación acrecienta las exportaciones netas, porque sube el precio relativo de los bienes importados y reduce el de las exportaciones nacionales en los mercados extranjeros. Este fortalecimiento de las exportaciones netas reales fomenta la demanda de bienes nacionales.

El efecto de balance puede tener cualquiera de los signos. Cuando la semielasticidad de la prima de riesgo con respecto a la relación entre la inversión y la deuda neta $(\iota)$ y la relación entre la deuda externa y el patrimonio neto de los capitalistas $(\chi)$ están por debajo de determinados umbrales se produce un resultado positivo, ligado al aumento de la demanda de producción nacional. El canal de política monetaria corresponde a la reacción normativa de las autoridades monetarias. $\mathrm{Su}$ aporte a la expansión de la demanda agregada de producción nacional se relaciona directamente con el peso asignado a las fluctuaciones del tipo de cambio en la función de pérdida del banco central $\left(W_{s}\right)$. Cuanto menor sea el valor de $W_{s}$, menos se permitirá la fluctuación del tipo de cambio, reduciendo sus efectos en la demanda agregada. Por último, para una determinada expansión de dicha demanda, los efectos finales en el nivel de precios internos dependen de la pendiente de la oferta agregada. Esto se relaciona directamente con la rigidez de los precios $(\theta)$, la que a su vez está directamente vinculada con la elasticidad de los precios de la curva de la oferta agregada $\left(\lambda_{\tilde{y}}\right)$.

Para obtener resultados empíricos se calibra el modelo y se derivan las respuestas de las variables endógenas a algunos shocks exógenos específicos. Para ello se asignan valores razonables a los parámetros en conformidad con la literatura pertinente. En el cuadro 1 se resumen los valores de referencia que se adoptaron para implementar los ejercicios empíricos y la fuente original en los casos correspondientes.

efecto inicial en el tipo de cambio nominal se transmite a continuación a otras variables, incluido el nivel de precios internos, siguiendo un proceso de interacciones recíprocas. 
CUADRO 1

Valores de los parámetros

\begin{tabular}{|c|c|c|}
\hline Parámetro & Valor & Información adicional \\
\hline $1<\theta<0$ & 0,75 & $\begin{array}{l}\text { Probabilidad de no ajustar el precio de la producción nacional (Fraga, } \\
\text { Goldfajn y Minella, 2003). }\end{array}$ \\
\hline$\beta<1$ & 0,99 & Factor de descuento intertemporal (Fraga, Goldfajn y Minella, 2003). \\
\hline $0<\alpha<1$ & 0,8 & Elasticidad producción-trabajo (Fraga, Goldfajn y Minella, 2003). \\
\hline $0<\eta<1$ & 0,2 & $\begin{array}{l}\text { Elasticidad exportaciones netas-tipo de cambio real (Fraga, Goldfajn y } \\
\text { Minella, 2003; Batini, Harrison y Millard, 2001). }\end{array}$ \\
\hline$\gamma_{c}>1$ & 2 & $\begin{array}{l}\text { Valor inverso de la elasticidad intertemporal del consumo (Céspedes y } \\
\text { Soto, 2005). }\end{array}$ \\
\hline$\gamma_{n}>1$ & 1 & $\begin{array}{l}\text { Valor inverso de la elasticidad del trabajo con respecto al salario real } \\
\text { (Céspedes y Soto, 2005). }\end{array}$ \\
\hline $0<\delta<1$ & 0,01 & $\begin{array}{l}\text { Proporción del comercio internacional del país en el comercio } \\
\text { internacional mundial. }\end{array}$ \\
\hline $0<\gamma<1$ & 0,6 & $\begin{array}{l}\text { Grado de preferencia por bienes nacionales en la canasta de consumo } \\
\text { (sesgo nacional) (Tovar, 2005; Céspedes y Soto, 2005). }\end{array}$ \\
\hline$W_{\tilde{y}}$ & 0,03 & $\begin{array}{l}\text { Peso asignado a la estabilización de la producción en la función de } \\
\text { pérdida del banco central. Este valor es superior al que figura en Fraga, } \\
\text { Goldfajn y Minella (2003). }\end{array}$ \\
\hline$W_{s}$ & 0,0014 & \\
\hline$W_{\pi}$ & 1 & \\
\hline$\iota$ & 0,51 & $\begin{array}{l}\text { Semielasticidad de la prima de riesgo con respecto a la relación entre } \\
\text { capital y valor neto (Tovar, 2005). }\end{array}$ \\
\hline$\chi=\frac{Q^{S S} D E^{S S}}{N E^{S S}}$ & 1,25 & $\begin{array}{l}\text { Relación entre deuda externa de los capitalistas y valor neto del estado } \\
\text { estacionario, medida en moneda nacional y términos nominales (Tovar, } \\
\text { 2005). }\end{array}$ \\
\hline
\end{tabular}

\begin{tabular}{lll}
\hline$s b r=\frac{\left(Q^{S S}\right)^{\gamma} B^{* S S}}{C^{S S}}>1$ & 0,63 & $\begin{array}{l}\text { Relación entre deuda externa y consumo privado en el estado } \\
\text { estacionario. Ambas variables se miden en moneda extranjera. }\end{array}$ \\
\hline $0<\operatorname{sir}=\frac{\left(Q^{S S}\right)^{1-\gamma} I^{S S}}{Y^{S S}}=<1$ & 0,2 & $\begin{array}{l}\text { Relación entre inversión privada y producción nacional en el estado } \\
\text { estacionario. Ambas variables se miden en moneda nacional. }\end{array}$ \\
$0<s_{x}=\frac{X^{S S}}{Y^{S S}}<1$ & 0,26 & $\begin{array}{l}\text { Proporción de las exportaciones en la producción nacional en el estado } \\
\text { estacionario. }\end{array}$
\end{tabular}

$0<s_{g}=\frac{G^{S S}}{Y^{S S}}<1 \quad 0,14 \quad \begin{aligned} & \text { Proporción del consumo del gobierno en la producción nacional en el } \\ & \text { estado estacionario. }\end{aligned}$

\begin{tabular}{ll}
\hline $0<s_{c}=\gamma s c r<1$ & $0,6 * 0,65=0,39$ \\
\hline $0<s_{\text {in }}=\gamma$ sir $<1$ & $0,6 * 0,2=0,12$
\end{tabular}

$0<s_{q}=(1-\gamma)\left(s_{c}+s_{\text {in }}\right)<1 \quad 0,4 *(0,39+0,12)=0,204$

$h_{y}=\frac{Y^{S S}}{C^{S S}}=\frac{1}{s c r}>1 \quad 1 / 0,65=1,538$

$0<h_{g}=\frac{G^{S S}}{C^{S S}}=\frac{s_{g}}{s c r}>1 \quad 0,14 / 0,65=0,215$ 
Cuadro 1 (conclusión)

\begin{tabular}{|c|c|}
\hline Parámetro & Información adicional \\
\hline$h_{q}=\gamma_{s} b r>0$ & $0,6^{*} 0,63=0,378$ \\
\hline$h_{b}{ }^{*}=s b r>0$ & 0,63 \\
\hline$\rho_{y} *<1$ & 0,90 \\
\hline$\rho_{\tilde{y}}^{*}<1$ & 0,90 \\
\hline$\rho_{\bar{y}} *<1$ & 0,98 \\
\hline$\rho_{\mu}<1$ & 0,95 \\
\hline$\rho_{a}<1$ & 0,95 \\
\hline$\rho_{b} *<1$ & 0,80 \\
\hline$\rho_{g}<1$ & 0,5 \\
\hline$\rho_{\phi}<1$ & 0,3 \\
\hline$\rho_{r} *<1$ & 0,5 \\
\hline$\rho_{r e}<1$ & 0,7 \\
\hline$\rho_{p} *<1$ & 0,9 \\
\hline$\rho_{d e}<1$ & 0,8 \\
\hline $\begin{array}{l}\rho_{s} T<1 \\
\rho_{\pi} T<1\end{array}$ & $\begin{array}{l}1 \\
1\end{array}$ \\
\hline$\rho_{\vartheta}<1$ & 0,8 \\
\hline
\end{tabular}

Fuente: elaboración propia.

\section{III}

\section{Los efectos de las perturbaciones externas}

En esta sección se calculan y representan gráficamente los efectos de los cambios en una desviación estándar de tres variables exógenas, $p_{t}^{*}, y_{t}^{*}$ y $r_{t}^{*}$, en las dos variables endógenas, $p_{t}^{H}$ y $S_{t}$. Más específicamente, se cuantifica el valor de las elasticidades proporcionadas por las fórmulas (A9) a (A14) (véase el anexo) para diferentes valores de los parámetros de interés. A fin de establecer la incidencia específica que tienen la rigidez del tipo de cambio (relacionada con $\left(W_{s}\right)$, el endeudamiento relativo de los capitalistas $(\chi)$, la sensibilidad de la prima de riesgo país a la inversión relativa $(\iota)$ y la rigidez de los precios internos $(\theta)$ en estos efectos, se realizan estimaciones utilizando valores alternativos para estos parámetros clave.

En el cuadro 2 se presentan los coeficientes estimados de traspaso, en tanto por uno, del tipo de cambio correspondientes a los tres shocks y para valores alternativos de los indicadores de rigidez del tipo de cambio, riesgo y endeudamiento, y la probabilidad de no ajustar los precios en el período actual. 
Coeficientes de traspaso del tipo de cambio $\left(p_{t}^{H} / s_{t}\right)$ correspondientes a shocks en las variables $p_{t}^{*}, y_{t}^{*} \mathbf{y} r_{t}^{*} \mathbf{y}$ valores alternativos de los parámetros, $\iota, \chi \mathbf{y} W_{s} \theta$

\begin{tabular}{lccccc}
\hline Shocks & $\begin{array}{c}\text { Puntos de referencia } \\
\text { para todos los } \\
\text { parámetros }\end{array}$ & $\begin{array}{c}\text { Puntos de referencia a } \\
\text { excepción de } \\
W_{s}=0,0015\end{array}$ & $\begin{array}{c}\text { Puntos de referencia } \\
\text { a excepción de } \\
\iota=1,5\end{array}$ & $\begin{array}{c}\text { Puntos de referencia } \\
\text { a excepción de } \\
\chi=2,0\end{array}$ & $\begin{array}{c}\text { Puntos de referencia } \\
\text { a excepción de } \\
\theta=0,8\end{array}$ \\
\hline$p_{t}^{*}$ & 0,88 & 0,95 & 0,81 & 0,88 & 0,96 \\
$y_{t}^{*}$ & 0,86 & 0,90 & 0,86 & 0,86 & 0,94 \\
$r_{t}^{*}$ & 0,36 & 0,39 & 0,36 & 0,36 & 0,37 \\
\hline
\end{tabular}

Fuente: elaboración propia a partir de las elasticidades derivadas del modelo: $W_{s}=0,0014, \iota=0,51, \chi=1,25, \theta=0,75, \gamma=0,6$.

En los gráficos 1, 2 y 3 se muestran los efectos de un aumento en el grado de rigidez del tipo de cambio. Se representan los resultados de las elasticidades de las dos variables endógenas con respecto al precio externo $\left(p_{t}^{*}\right)$, la producción externa $\left(y_{t}^{*}\right)$ y la tasa de interés real externa $\left(r_{t}^{*}\right)$, respectivamente. En cada gráfico se presentan los resultados para dos valores alternativos de la rigidez del tipo de cambio, a saber: $W_{s}=0,0014$ y $W_{s}=0,0015$.

Los gráficos 1 a 3 y las estimaciones numéricas presentadas en la tercera columna del cuadro 2 reflejan algunos resultados comunes. En primer lugar, el impacto de los shocks es mayor en el tipo de cambio que en los precios internos, lo que confirma que el traspaso del tipo de cambio es solo parcial en el corto plazo. Por otra parte, los efectos más intensos en las dos variables endógenas corresponden a los que produce la variación de la producción externa. En segundo lugar, cuando la economía resulta afectada debido a incrementos en la producción y en los precios externos, la mayor rigidez del tipo de cambio nominal restringe las dos variables endógenas (el tipo de cambio y el nivel de precios internos), pero aumenta el traspaso del tipo de cambio. Cuando la curva de Phillips se aplana, se obtienen resultados similares. Sin embargo, cuando el shock externo consiste en un incremento de la tasa de interés real externa, una mayor flexibilidad del tipo de cambio y curvas de Phillips más verticales amortiguan la respuesta de las dos variables endógenas, pero sin alterar el traspaso del tipo de cambio.

GRÁFICO 1

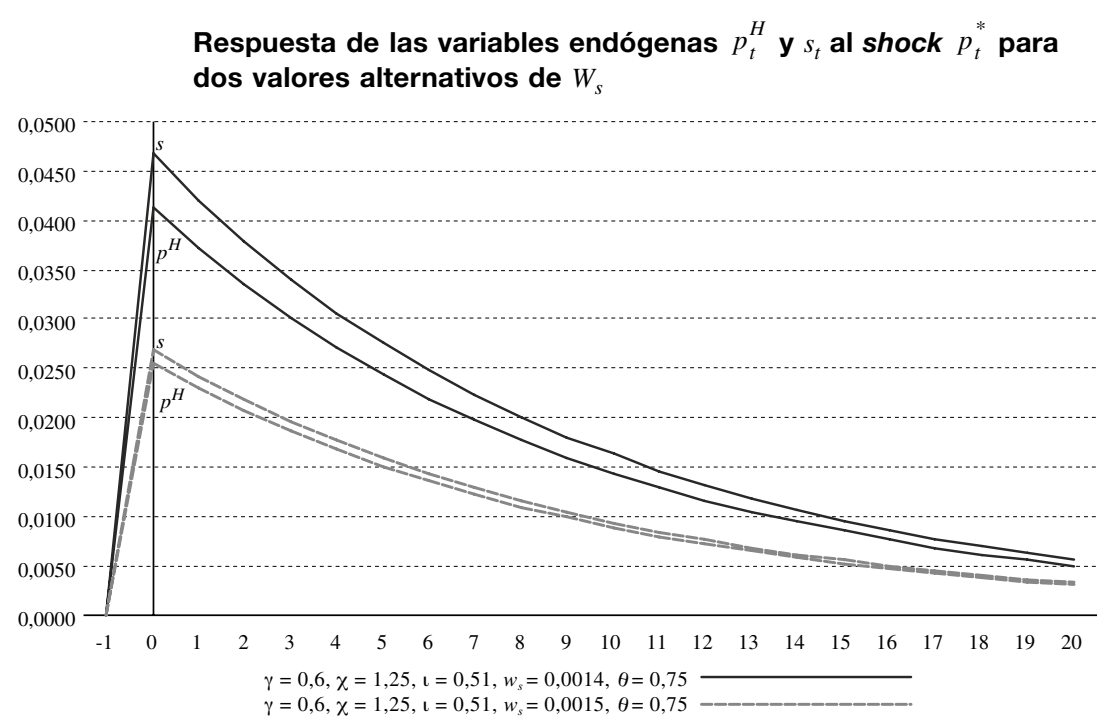

Fuente: elaboración propia a partir de las elasticidades derivadas del modelo. 
GRÁFICO 2

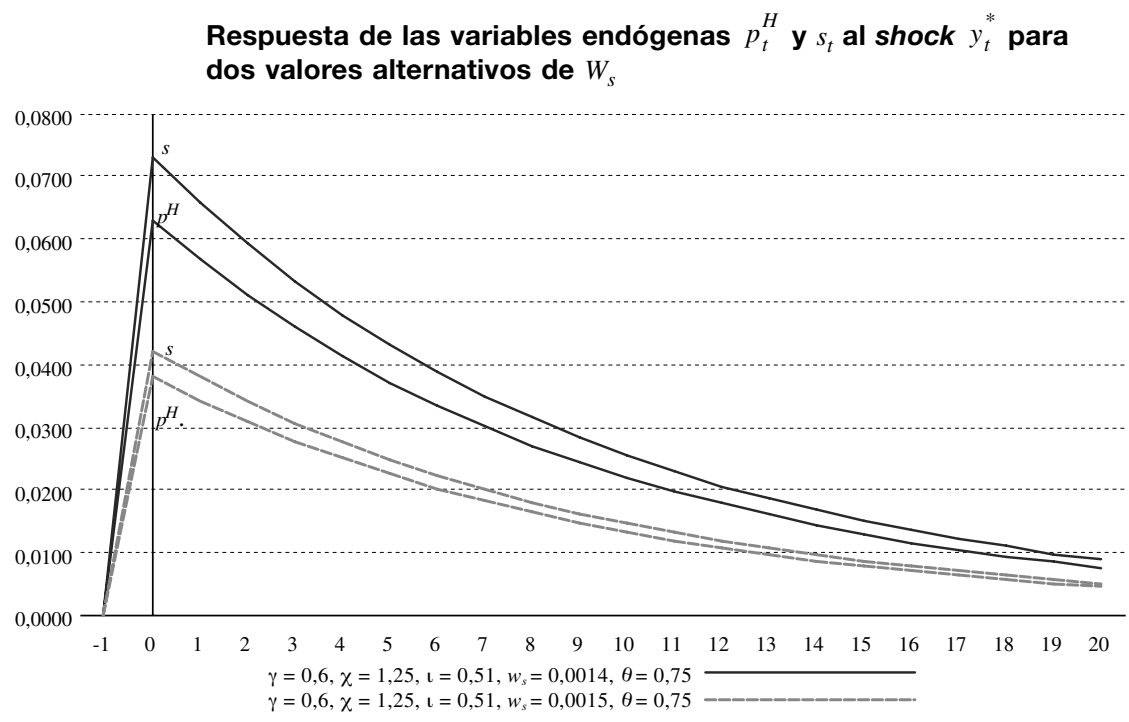

Fuente: elaboración propia a partir de las elasticidades derivadas del modelo.

GRÁFICO 3

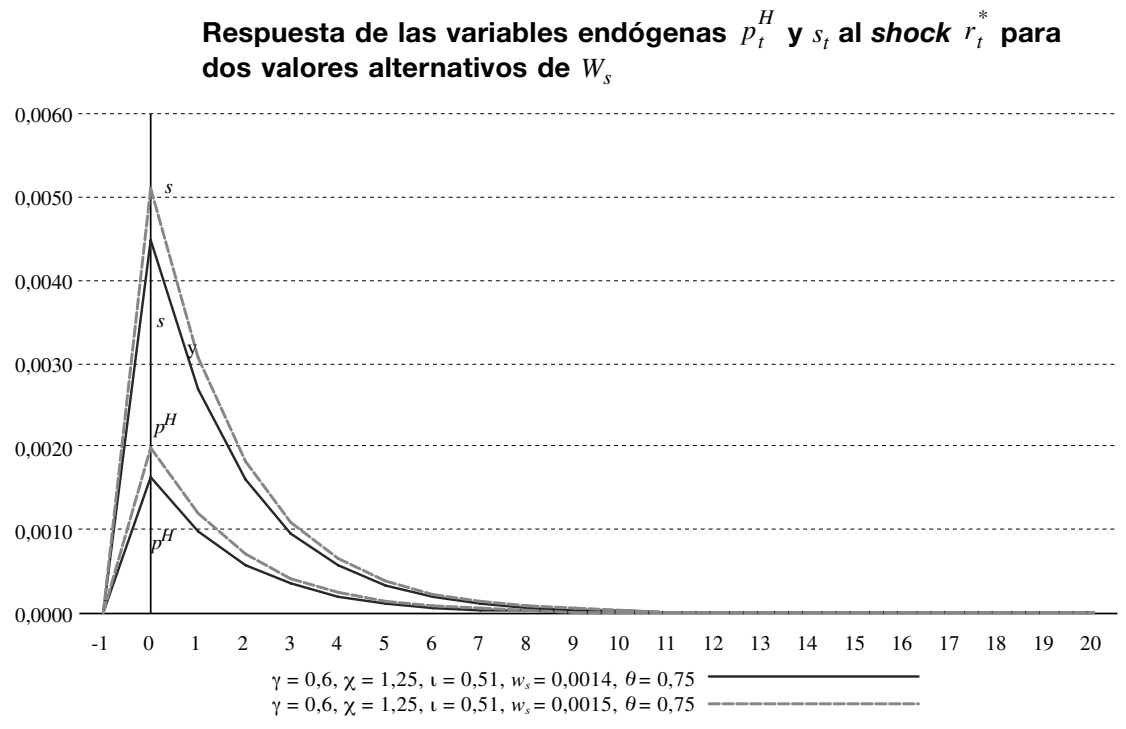

Fuente: elaboración propia a partir de las elasticidades derivadas del modelo.

En las actuales circunstancias de debilidad económica y elevadas tasas de desempleo, muchos bancos centrales han asignado mayor peso a la estabilización de la producción $\left(W_{y}\right)$ que a la estabilización del tipo de cambio $\left(W_{s}\right)$ en su función de pérdida social, con el objeto de atenuar las fluctuaciones en la producción interna real y el empleo. De acuerdo con los resultados, este cambio de política también contribuirá a reducir el traspaso del tipo de cambio.

En los gráficos 4 a 6 se muestran las elasticidades relacionadas con cada shock para dos valores alternativos de la sensibilidad de la prima de riesgo país a la inversión relativa ( $\iota$. Se observa una vez más que la reacción del tipo de cambio nominal es en cada caso más marcada que 
la del nivel de precios internos. Asimismo, un aumento en $\iota$ agranda la reacción tanto del tipo de cambio nominal como del nivel de precios internos, sin cambiar el traspaso de la tasa de cambio. Esto se aplica a los ajustes causados por cualquier tipo de shock externo.

No obstante, los efectos de un incremento de la tasa de interés real externa en el tipo de cambio nominal y en el nivel de precios internos (véase el gráfico 6) son contraintuitivos para niveles relativamente altos de $\iota$. En efecto, de acuerdo con variaciones del modelo MundellFleming, un aumento de la tasa de interés externa provoca salidas de capital que deprecian la moneda nacional, estimulando de ese modo la producción nacional. En el modelo utilizado en este trabajo, un incremento de $r_{t}^{*}$ acrecienta el costo internacional del capital, lo que reduce la inversión interna (ecuación (6)) porque los capitalistas se endeudan en el exterior para financiar sus gastos de inversión. El descenso de la inversión aminora la prima de riesgo. En el caso de los valores de referencia de $\iota$, $\chi$ y $\eta$ asumidos en la presente calibración, el aumento de la tasa de interés externa es mayor que el impacto en la prima de riesgo, dando lugar por consiguiente a reacciones normales del tipo de cambio nominal y del nivel de precios internos. Sin embargo, si la prima de riesgo disminuye más de lo que engrosa la tasa de interés externa, la afluencia de capital financiero incrementa el tipo de cambio nominal. La apreciación del tipo de cambio debilita la demanda de bienes nacionales, causando la reducción de los precios y de la producción nacionales. Esos resultados "anormales" se muestran en el gráfico 6, donde se asume que el parámetro de riesgo $(\iota$,$) aumenta de manera suficiente. Por último, el banco$ central reacciona bajando la tasa de interés nominal para frenar las fluctuaciones del tipo de cambio nominal y de la producción. Cabe destacar que estas reacciones anormales a los shocks en la tasa de interés real externa tienen lugar solo cuando los valores de $\iota$ superan un determinado umbral.

GRÁFICO 4

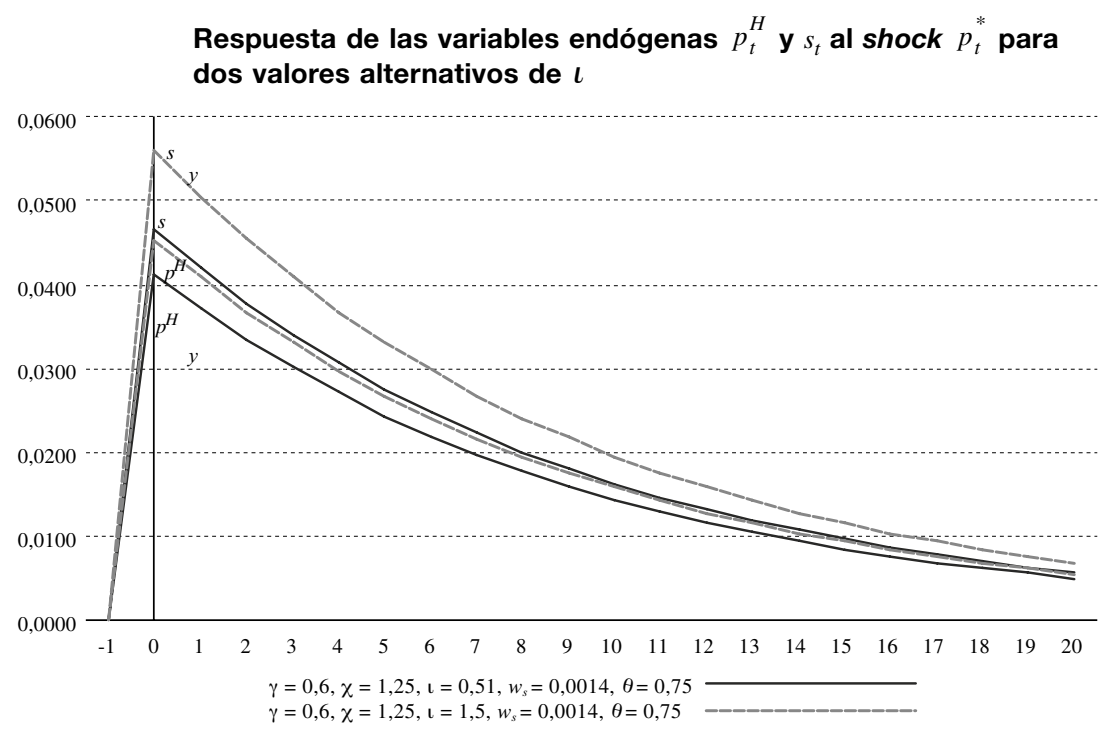

Fuente: elaboración propia a partir de las elasticidades derivadas del modelo. 
GRÁFICO 5

Respuesta de las variables endógenas $p_{t}^{H}$ y $s_{t}$ al shock $y_{t}^{*}$ para dos valores alternativos de $\iota$

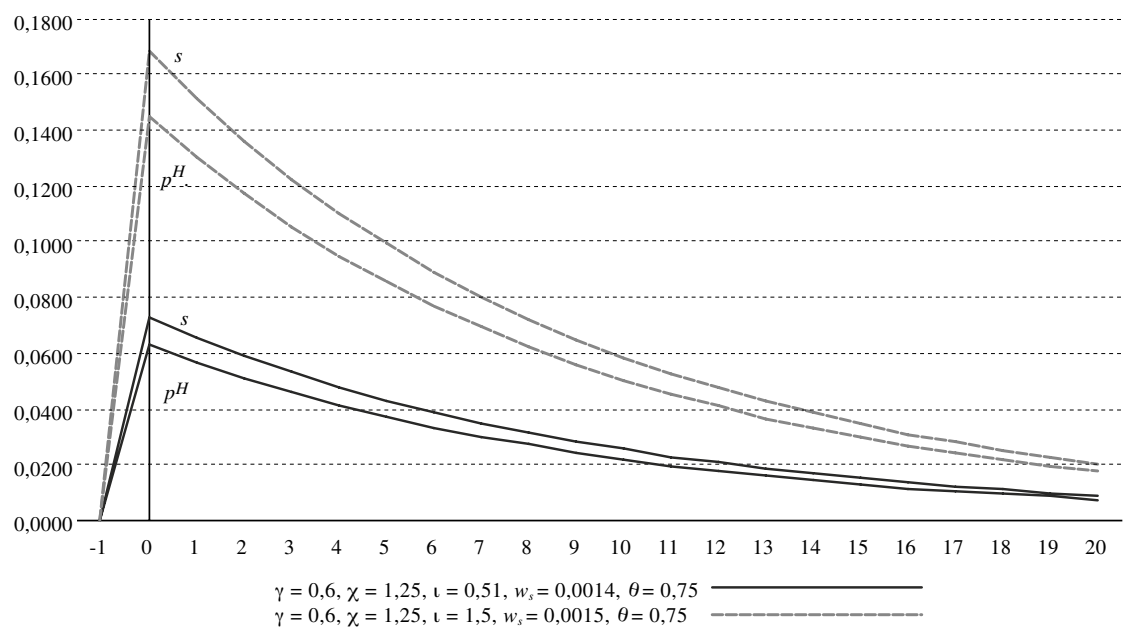

Fuente: elaboración propia a partir de las elasticidades derivadas del modelo.

GRÁFICO 6

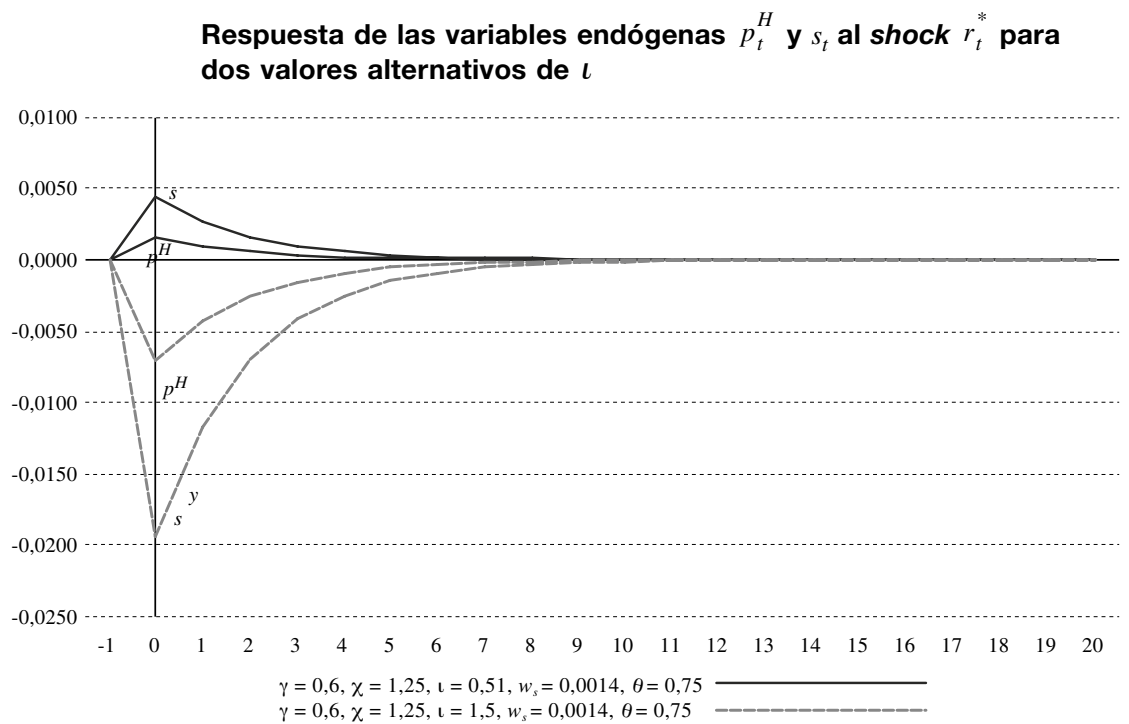

Fuente: elaboración propia a partir de las elasticidades derivadas del modelo.

En los gráficos 7 a 9 se representan las elasticidades correspondientes a los tres shocks y a dos valores alternativos del endeudamiento relativo de los capitalistas $(\chi)$. Como en los casos anteriores, la reacción del tipo de cambio nominal es más intensa que la del nivel de precios internos después de cada shock. Asimismo, el incremento de $\chi$ no modifica el traspaso del tipo de cambio bruto. 
GRÁFICO 7

Respuesta de las variables endógenas $p_{t}^{H}$ y $s_{t}$ al shock $p_{t}^{*}$ para dos valores alternativos de $\chi$

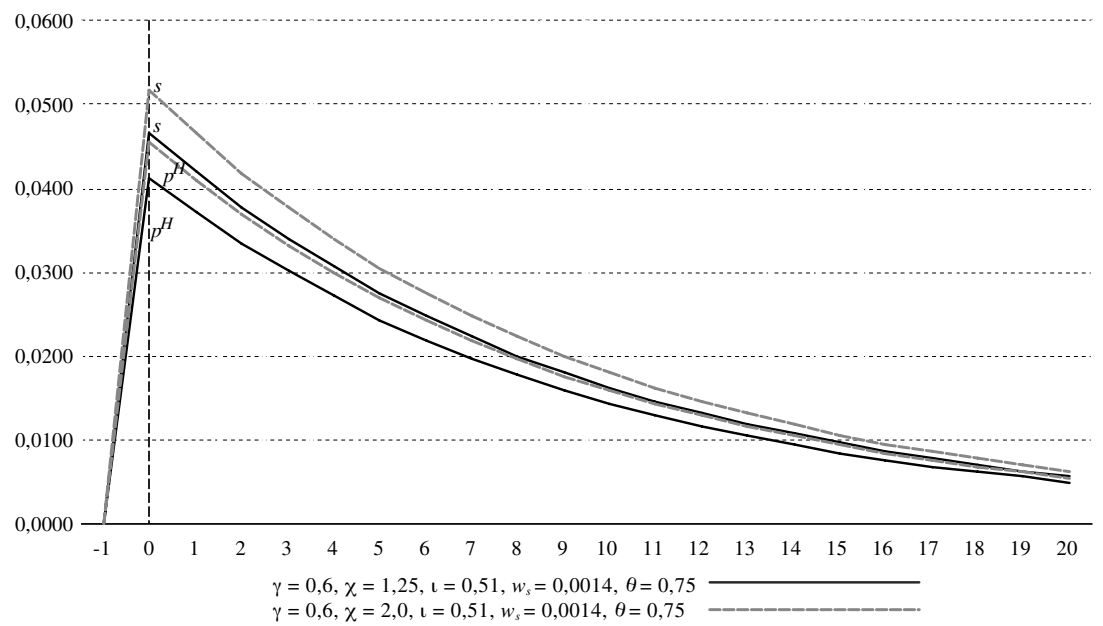

Fuente: elaboración propia a partir de las elasticidades derivadas del modelo.

GRÁFICO 8

Respuesta de las variables endógenas $p_{t}^{H}$ y $s_{t}$ al shock $y_{t}^{*}$ para dos valores alternativos de $\chi$

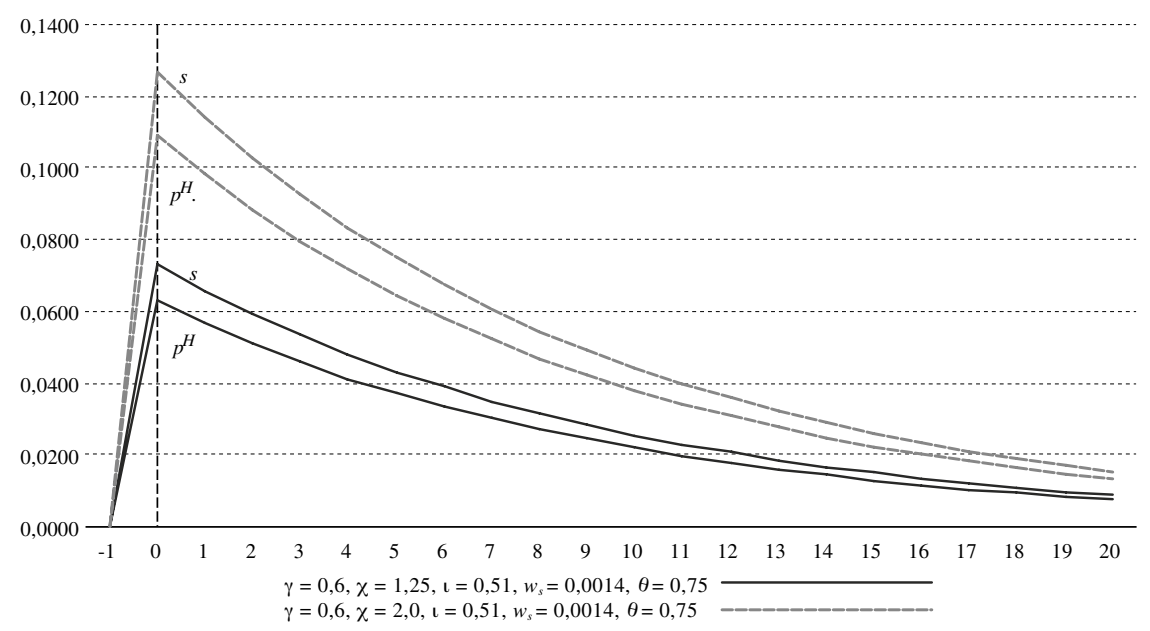

Fuente: elaboración propia a partir de las elasticidades derivadas del modelo. 
GRÁFICO 9

Respuesta de las variables endógenas $p_{t}^{H}$ y $s_{t}$ al shock $r_{t}^{*}$ para dos valores alternativos de $\chi$

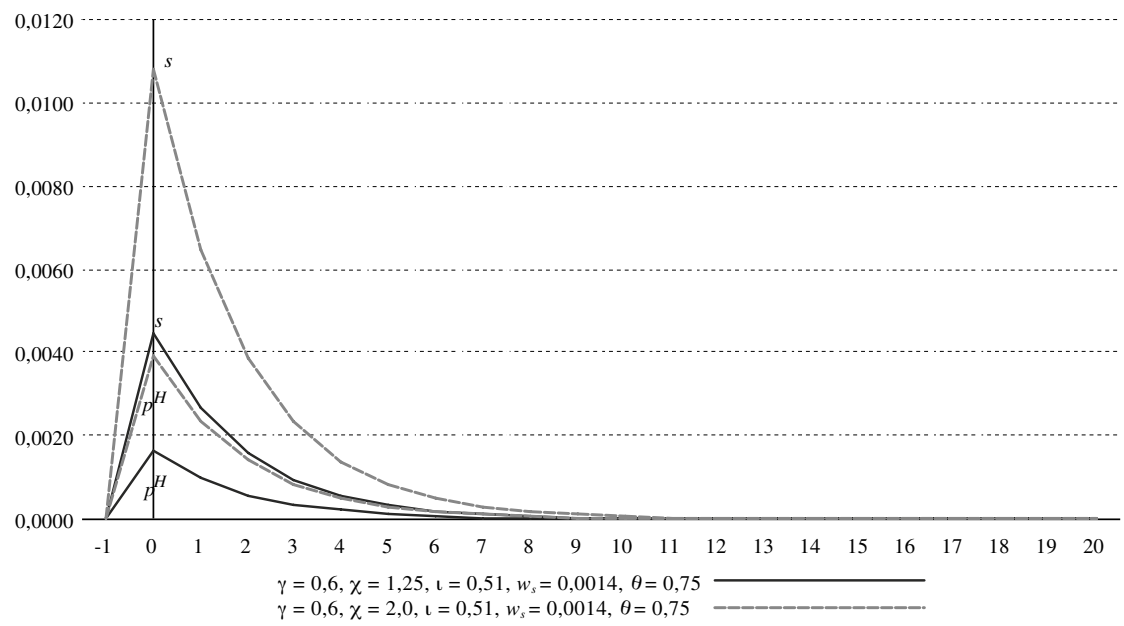

Fuente: elaboración propia a partir de las elasticidades derivadas del modelo.

Por último, en los gráficos 10 a 12 se presentan los resultados relativos a los tres shocks con respecto a la probabilidad de no ajustar los precios en el período actual $\theta$. Como puede apreciarse, cuando los shocks que recibe la economía consisten en elevaciones de los precios y de la producción extranjeros, se obtiene un incremento de 0,75 a 0,80 en la rigidez de los precios que amortigua la depreciación del tipo de cambio nominal y el aumento de los precios internos, mientras que incrementa el traspaso del tipo de cambio. Sin embargo, cuando la economía resulta afectada por una tasa de interés real externa más alta, las curvas de Phillips más planas exacerban las reacciones de las dos variables endógenas sin alterar el traspaso del tipo de cambio.

GRÁFICO 10

Respuesta de las variables endógenas $p_{t}^{H} \mathbf{y} s_{t}$ al shock $p_{t}^{*}$ para dos valores alternativos de $\theta$

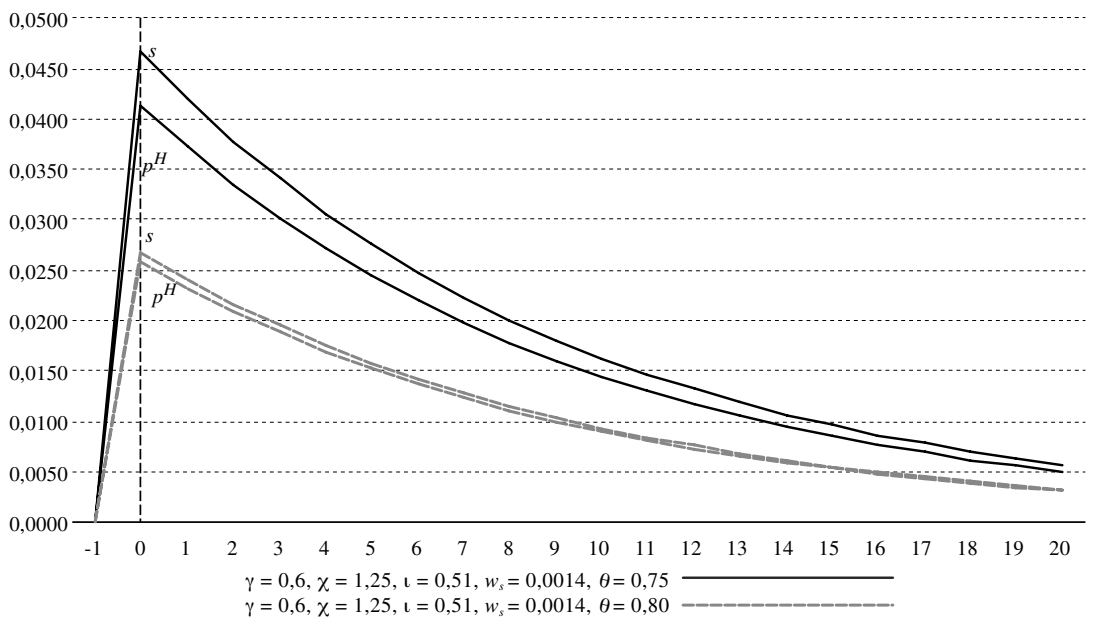

Fuente: elaboración propia a partir de las elasticidades derivadas del modelo. 
GRÁFICO 11

Respuesta de las variables endógenas $p_{t}^{H}$ y $s_{t}$ al shock $y_{t}^{*}$ para dos valores alternativos de $\theta$

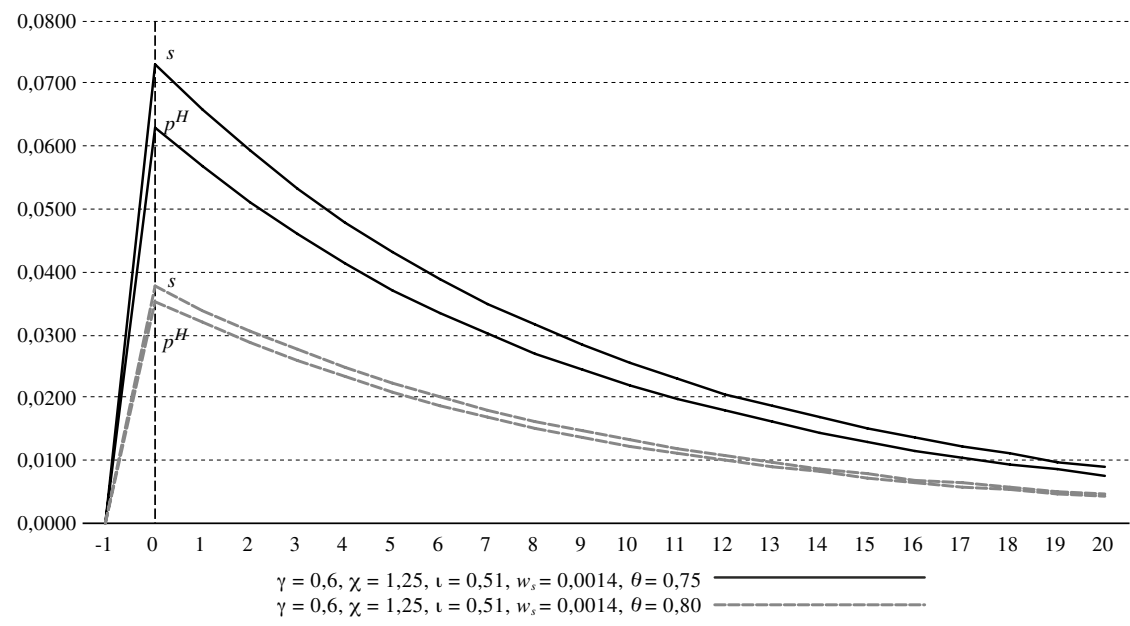

Fuente: elaboración propia a partir de las elasticidades derivadas del modelo.

GRÁFICO 12

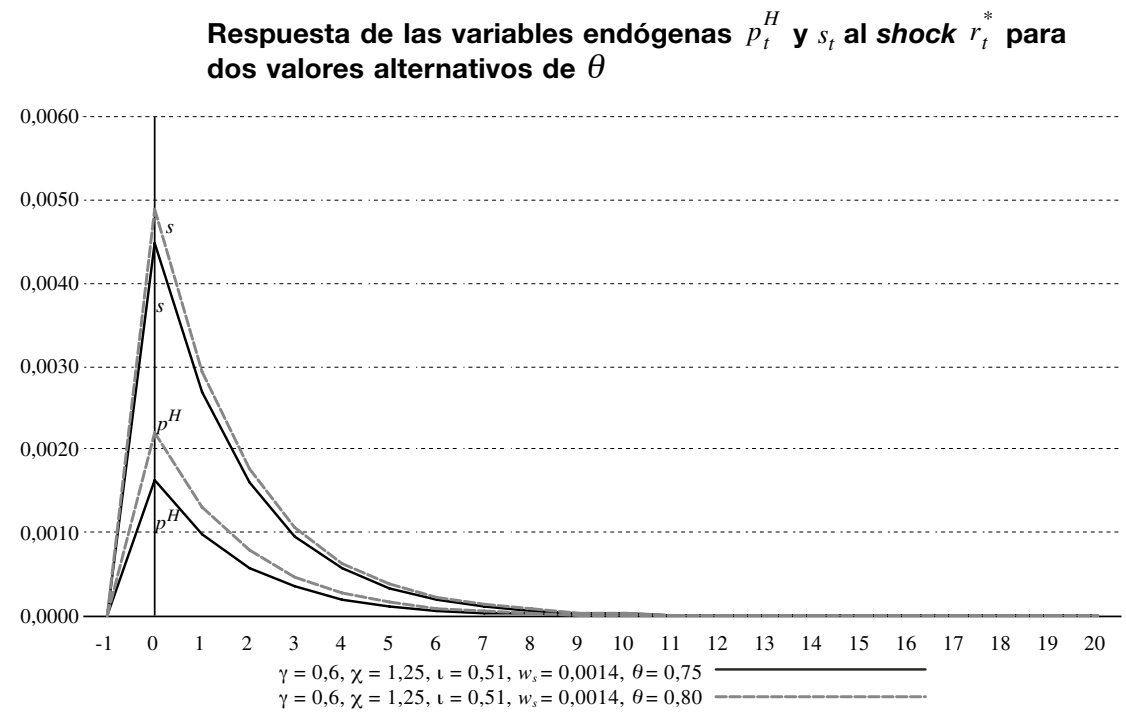

Fuente: elaboración propia a partir de las elasticidades derivadas del modelo.

Los resultados relativos a las variaciones en $\iota \mathrm{y} \chi$ son similares a los de Nogueira y León-Ledesma (2011), que utilizan el índice de bonos de mercados emergentes (EMBI+) y el diferencial de la tasa de interés real con los Estados Unidos de América como sustitutos de las percepciones del mercado de la condición económica nacional. En ambos casos, la inestabilidad macroeconómica y el aumento del riesgo país conducen a mayores fluctuaciones del tipo de cambio y del nivel de precios internos, y al mismo tiempo incrementan el traspaso del tipo de cambio bruto. En consecuencia, los resultados del presente trabajo también respaldan indirectamente la opinión general de que los ambientes macroeconómicos favorables, incluidos aquellos en los que los bancos centrales aplican regímenes de metas de inflación, contribuyen en forma decisiva a controlar la volatilidad de los precios internos y el tipo de cambio, como también a disminuir el grado de traspaso del tipo de cambio bruto. 


\section{IV}

\section{Conclusiones}

En este trabajo se construyó y aplicó un modelo macroeconómico de equilibrio general para evaluar la sensibilidad del tipo de cambio nominal y el nivel de precios internos ante tres tipos de shocks exógenos y calcular el consiguiente impacto en el traspaso del tipo de cambio bruto en las economías emergentes. El modelo incluye dos elementos necesarios para abordar correctamente el tema en este tipo de economías. El primero es el grado de flexibilidad del tipo de cambio en la función de pérdida social, que se necesita para evaluar la influencia de la flexibilidad en el traspaso del tipo de cambio. El segundo es la inclusión de parámetros de balance en el canal de transmisión de efectos del traspaso del tipo de cambio. Asimismo, las propiedades de equilibrio general del modelo permitieron verificar la proposición de Mishkin (2008), según la cual el grado de traspaso del tipo de cambio depende en gran medida del tipo de shock externo que afecta a la economía.

Se obtuvieron tres resultados significativos. En primer lugar, la rigidez del tipo de cambio disminuye la volatilidad de las dos variables endógenas y aumenta el coeficiente de traspaso del tipo de cambio cuando la economía resulta afectada por variaciones en la producción y el nivel de precios externos. Sin embargo, la rigidez del tipo de cambio exacerba las fluctuaciones de las dos variables endógenas cuando el shock se debe a variaciones en la tasa de interés real externa. En segundo lugar, el incremento de las fuentes de inestabilidad macroeconómica, como la sensibilidad de la prima de riesgo país a la inversión relativa y el endeudamiento relativo de los capitalistas, también acrecienta la volatilidad de las dos variables endógenas, aunque no afecta al grado de traspaso del tipo de cambio bruto. Estos resultados confirman los descritos previamente en la literatura. En tercer lugar, cuando los shocks que recibe la economía consisten en variaciones en la producción y el nivel de precios externos, las curvas de Phillips más planas - como las que se observan en muchas economías en el contexto de crisis económica actual—ayudan a minimizar los ajustes del tipo de cambio nominal y el nivel de precios internos, en tanto que aumentan el traspaso del tipo de cambio.

De los resultados derivan algunas repercusiones desde el punto de vista normativo. En primer término, las autoridades de las economías emergentes que desean reducir el grado de traspaso del tipo de cambio (para controlar la inflación o hacer más eficaz la política monetaria), deberían considerar seriamente las ventajas macroeconómicas que proporcionan la flexibilidad del tipo de cambio y un ambiente macroeconómico estable (donde no predominan el endeudamiento y el riesgo económico). Por su parte, visto que esos elementos constituyen importantes pilares de los regímenes de metas de inflación, los resultados obtenidos en este trabajo confirman indirectamente que el establecimiento de metas de inflación estabiliza los tipos de cambio y los precios internos, potenciando los efectos macroeconómicos positivos de un régimen de ese tipo en las economías emergentes, como se demuestra en algunas obras de la literatura reciente ${ }^{9}$. Si bien las medidas de los bancos centrales tendientes a estabilizar la inflación bajo curvas de Phillips más planas podrían perjudicar el crecimiento económico, estos pueden abordar el problema aumentando el peso de la estabilización de la producción en sus funciones de pérdida social ${ }^{10}$.

\footnotetext{
9 Véanse, por ejemplo, Vega y Winkelried (2005), y García-Solanes y Torrejón-Flores (2012).

${ }^{10}$ En FMI (2013) se mencionan otras maneras de abordar este problema.
} 
ANEXO

\section{Resolución matemática del modelo}

Para resolver el modelo, primeramente se deriva la ecuación que especifica el valor de equilibrio de $p_{t}^{H E}$ utilizando el método de los coeficientes indeterminados. El procedimiento es el siguiente: se minimiza la función de pérdida (1) sujeto a la oferta agregada (2) para derivar las trayectorias óptimas de la brecha de producción interna y el tipo de cambio nominal:

$$
\begin{gathered}
\tilde{y}_{t}=\frac{\lambda_{\tilde{y}} w_{s} w_{\pi}}{w_{\tilde{y}}\left(w_{s}+\lambda_{q}^{2} w_{\pi}\right)+\lambda_{\tilde{y}}^{2} w_{s} w_{\pi}}\left[-\lambda_{r e} r e_{t}-\lambda_{q} p_{t}^{*}+\lambda_{q} p_{t}^{H}-\lambda_{\tilde{y}} \tilde{y}^{*}-\beta E_{t}\left(\pi_{t+1}^{H}\right)-\mu_{t}+\pi_{t}^{H T}-\lambda_{q} s_{t}^{T}\right] \\
s_{t}=\frac{\lambda_{q} w_{\tilde{y}} w_{\pi}}{w_{\tilde{y}}\left(w_{s}+\lambda_{q}^{2} w_{\pi}\right)+\lambda_{\tilde{y}}^{2} w_{s} w_{\pi}}\left[-\lambda_{r e} r e_{t}-\lambda_{q} p_{t}^{*}+\lambda_{q} p_{t}^{H}-\lambda_{\tilde{y}} \tilde{y}^{*}-\beta E_{t}\left(\pi_{t+1}^{H}\right)-\mu_{t}+\pi_{t}^{H T}+\frac{w_{s}\left(w_{\tilde{y}}+\lambda_{\tilde{y}}^{2} w_{\pi}\right)}{\lambda_{q} w_{\tilde{y}} w_{\pi}} s_{t}^{T}\right]
\end{gathered}
$$

A continuación se sustituye la prima de riesgo (5) en la paridad descubierta de la tasa de interés (4), y se inserta el resultado en la demanda agregada (3). Luego se combina la expresión resultante con la ecuación BP (7) y se resuelve para $y_{t}=\tilde{y}_{t}+\bar{y}_{t}$. Se insertan (A1) y (A2) en la expresión resultante y se resuelve para $E_{t}\left(p_{t+2}^{H}\right)$.

Se obtiene:

$$
\begin{gathered}
E_{t}\left(p_{t+2}^{H}\right)=d_{1} E_{t}\left(p_{t+1}^{H}\right)+d_{0} p_{t}^{H}+C_{y^{*}} y_{t}^{*}+C_{\bar{y}^{*}} \bar{y}_{t}^{*}+C_{\mu} \mu_{t}+C_{a} a_{t}+C_{b^{*}} b_{t}^{*}+C_{g} g_{t}+C_{\phi} \phi_{t}+C_{r^{*}} r_{t}^{*} \\
+C_{r e} r e_{t}+C_{p^{*}} p_{t}^{*}+C_{d e} d e_{t}+C_{s} s_{t}^{T}+C_{\pi^{H T}} \pi_{t}^{H T}+C_{\vartheta} \vartheta_{t}
\end{gathered}
$$

Se define:

$$
\begin{gathered}
p_{t}^{H}=B_{y^{*}} y_{t}^{*}+B_{\bar{y}^{*}} \bar{y}_{t}^{*}+B_{\mu} \mu_{t}+B_{a} a_{t}+B_{b^{*}} b_{t}^{*}+B_{g} g_{t}+B_{\phi} \phi_{t}+B_{r^{*}} r_{t}^{*} \\
+B_{r e} r e_{t}+B_{p^{*}} p_{t}^{*}+B_{d e} d e_{t}+B_{s} s_{t}^{T}+B_{\pi^{H T}} \pi_{t}^{H T}+B_{\vartheta} \vartheta_{t} \\
E_{t}\left(p_{t+1}^{H}\right)=B_{y^{*}} \rho_{y^{*}} y_{t}^{*}+B_{\bar{y}^{*}} \rho_{\bar{y}^{*}} \bar{y}_{t}^{*}+B_{\mu} \rho_{\mu} \mu_{t}+B_{a} \rho_{a} a_{t}+B_{b^{*}} \rho_{b^{*}} b_{t}^{*}+B_{g} \rho_{g} g_{t}+B_{\phi} \rho_{\phi} \phi_{t}+B_{r^{*}} \rho_{r^{*}} r_{t}^{*} \\
+B_{r e} \rho_{r e} r e_{t}+B_{p^{*}} \rho_{p^{*}} p_{t}^{*}+B_{d e} \rho_{d e} d e_{t}+B_{s} s_{t}^{T}+B_{\pi^{H T}} \pi_{t}^{H T}+B_{\vartheta} \rho_{\vartheta} \vartheta_{t}
\end{gathered}
$$


Se sustituyen las ecuaciones (A4) a (A6) en (A3). Al aplicar la metodología de los coeficientes indeterminados se derivan los coeficientes de $p_{t}^{H E}\left(B_{j}\right)$, con:

$$
B_{j}=\frac{C_{j}}{\rho_{j}^{2}-d_{1} \rho_{j}-d_{0}}
$$

donde $j=y^{*}, \bar{y}^{*}, \mu, a, b^{*}, g, \phi, r^{*}, r e, p^{*}, d e, \vartheta$.
$\operatorname{Para} s^{T} \mathrm{y} \pi^{H T}, B_{j}=C_{j} /\left[1-d_{1}-d_{0}\right]$.

Por ejemplo, $B_{y^{*}}$ es la elasticidad del precio interno con respecto a la producción externa. Con esta información, al aplicar los valores paramétricos (véase el cuadro 1) que se explican a continuación, se derivan los signos de las derivadas parciales en (A7):

$$
\begin{aligned}
& p_{t}^{H E}=B_{y^{*}} y_{t}^{*}+B_{\bar{y}} \bar{y}_{t}^{*}+B_{\mu} \mu_{t}+B_{a} a_{t}+B_{b^{*}} b_{t}^{*}+B_{g} g_{t}+B_{\phi} \phi_{t}+B_{r^{*}} r_{t}^{*} \\
& +\quad-\quad+\quad-\quad-\quad+\quad+\quad-1+ \\
& \begin{array}{c}
+B_{r e} r e_{t}+B_{p^{*}} p_{t}^{*}+B_{d e} d e_{t}+B_{s}{ }^{T} s_{t}^{T}+B_{\pi^{H T}} \pi_{t}^{H T}+B_{\vartheta} \vartheta_{t} \\
+\quad+ \\
+
\end{array}
\end{aligned}
$$

Para derivar la ecuación de equilibrio del tipo de cambio se aplica un procedimiento similar al del nivel de precios internos. En consecuencia,

$$
\begin{aligned}
& E_{t}\left(s_{t+1}\right)=d_{0}^{\prime} s_{t}+C^{\prime} y^{*} y_{t}^{*}+C^{\prime}{ }^{\prime}{ }^{*} \bar{y}_{t}^{*}+C^{\prime}{ }_{\mu} \mu_{t}+C^{\prime}{ }_{a} a_{t}+C_{b^{*}}^{\prime} b_{t}^{*}+C_{g}^{\prime} g_{t}+C^{\prime}{ }_{\phi} \phi_{t}+C^{\prime}{ }^{*}{ }^{*} r_{t}^{*} \\
& +C^{\prime}{ }_{r e} r e_{t}+C^{\prime}{ }_{p}{ }^{*} p_{t}^{*}+C^{\prime}{ }_{d e} d e_{t}+C^{\prime}{ }^{\prime} s_{t}^{T}+C^{\prime} \pi^{H T} \pi_{t}^{H T}+C^{\prime}{ }_{\vartheta} \vartheta_{t}
\end{aligned}
$$

Se derivan los coeficientes de $s_{t}^{H E}\left(S_{j}\right)$, con:

$$
S_{j}=\frac{C_{j}^{\prime}}{\rho_{j}-d_{0}^{\prime}}
$$

donde $j=y^{*}, \bar{y}^{*}, \mu, a, b^{*}, g, \phi, r^{*}, r e, p^{*}, d e, \vartheta$.

$$
\text { Para } s^{T} \mathrm{y} \pi^{H T}, S_{j}=C^{\prime}{ }_{j} /\left[1-d_{0}^{\prime}\right] \text {. }
$$

El resultado es:

$$
\begin{aligned}
& s_{t}^{E}=S_{y} y_{t}^{*}+S_{\bar{y}}^{*} \bar{y}_{t}^{*}+S_{\mu} \mu_{t}+S_{a} a_{t}+S_{b} b_{t}^{*}+S_{g} g_{t}+S_{\phi} \phi_{t}+S_{r} r_{t}^{*} \\
& +\quad-\quad-\quad-\quad-\quad+\quad+\quad-1+ \\
& +S_{r e} r e_{t}+S_{p^{*}} p_{t}^{*}+S_{d e} d e_{t}+S_{s} S_{t}^{T}+S_{\pi^{H T}} \pi_{t}^{H T}+S_{\vartheta} \vartheta_{t}
\end{aligned}
$$

$\mathrm{Al}$ tener en cuenta las ecuaciones que especifican los valores de equilibrio de las variables endógenas, es fácil derivar la elasticidad de cada variable endógena con respecto a las tres variables exógenas de interés, $p_{t}^{*}, y_{t}^{*} \mathrm{y} r_{t}^{*}$ :

$$
\begin{gathered}
B_{p^{*}}=\frac{\frac{1}{A}\left[\lambda_{\tilde{y}} \lambda_{q} w_{s} w_{\pi}\left(P-R \rho_{p^{*}}\right)-\lambda_{q}^{2} w_{\tilde{y}} w_{\pi}\left(M+N \rho_{p^{*}}\right)\right]+M+N \rho_{p^{*}}}{F_{p^{*}}} \\
B_{y^{*}}=\frac{\frac{1}{A}\left[\lambda_{\tilde{y}} \lambda_{\tilde{y}^{*}} w_{s} w_{\pi}\left(P-R \rho_{y^{*}}\right)-\lambda_{\tilde{y}} \lambda_{q} w_{\tilde{y}} w_{\pi}\left(M+N \rho_{y^{*}}\right)\right]+s_{x}}{F_{y^{*}}}
\end{gathered}
$$




$$
\begin{gathered}
B_{r^{*}}=\frac{-\left[\frac{s_{c}}{\gamma_{c}}+\frac{B}{(1+\iota)}\right]}{F_{r^{*}}} \\
S_{p^{*}}=\frac{\frac{\lambda_{\tilde{y}} w_{s} w_{\pi}}{A}\left\{B_{p^{*}}\left[\beta\left(1-\rho_{p^{*}}\right)+\lambda_{q}\right]-\lambda_{q}\right\}\left(R \rho_{p^{*}}-P\right)+\left(1-B_{p^{*}}\right)(N+M)}{-N \rho_{p^{*}}-M} \\
S_{y^{*}}=\frac{\frac{\lambda_{\tilde{y}} w_{s} w_{\pi}}{A}\left\{B_{y^{*}}\left[\beta\left(1-\rho_{y^{*}}\right)+\lambda_{q}\right]-\lambda_{\tilde{y}^{*}}\right\}\left(R \rho_{y^{*}}-P\right)-B_{y^{*}}\left(N \rho_{y^{*}}+M\right)+s_{x}}{-N \rho_{y^{*}}-M} \\
\frac{\lambda_{\tilde{y}} w_{s} w_{\pi}}{A}\left\{B_{r^{*}}\left[\beta\left(1-\rho_{r^{*}}\right)+\lambda_{q}\right]\right\}\left(R \rho_{r^{*}}-P\right)-\left[\frac{s_{c}}{\gamma_{c}}+\frac{B}{(1+\iota)}\right] \\
-N \rho_{r^{*}}-M
\end{gathered}
$$

donde:

$$
\begin{gathered}
F_{j}=R R \rho_{j}^{2}-\frac{1}{A}\left\{P \beta \lambda_{y} w_{s} w_{\pi}+\left(\beta+\lambda_{q}\right)\left(R \lambda_{y} w_{c} w_{s} w_{\pi}+N \lambda_{q} w_{y} w_{\pi}\right)\right. \\
\left.-M \beta \lambda_{q} w_{y} w_{\pi}\right\} \rho_{j}+N \rho_{j}-\frac{1}{A}\left\{\left(\beta+\lambda_{q}\right)\left(M \lambda_{q} w_{y} w_{\pi}-P \lambda_{y} w_{s} w_{\pi}\right)\right\}+M \\
j=y^{*}, r^{*} \mathrm{y} p^{*} .
\end{gathered}
$$

Además:

$$
\begin{gathered}
B=\left[s_{i n}-\frac{s_{c}}{\gamma_{c}} \iota\right] \\
A=w_{\tilde{y}}\left(w_{s}+\lambda_{q}^{2} w_{\pi}\right)+\lambda_{\tilde{y}}^{2} w_{s} w_{\pi} \\
R=s_{c} h_{y}+\frac{B}{(1+\iota)} \\
N=s_{c} h_{q}-\frac{s_{c}}{\gamma_{c}} \psi-B \frac{1+(1-\gamma)(1-\delta)}{1+\iota} \\
\left.M=\frac{s_{c}}{\gamma_{c}} \psi-\frac{s_{c}}{\gamma_{c}} \iota(1-\gamma)+\chi\right]+\left(s_{q}+s_{x} \eta\right)+B \frac{[1-(1-\gamma) \delta]-\iota[(1-\gamma)+\chi]}{1+\iota} \\
R R=\frac{\beta}{A}\left[R \lambda_{\tilde{y}} w_{s}+N w_{\tilde{y}} \lambda_{q}\right]
\end{gathered}
$$




$$
\begin{gathered}
P=1-\iota(\chi+1)\left[\frac{s_{c}}{\gamma_{c}}+\frac{B}{(1+\iota)}\right] \\
M_{1}=\frac{1+(1-\gamma)(1-\delta)}{1+\iota} \\
M_{2}=\frac{[1-(1-\gamma) \delta]-\iota[(1-\gamma)+\chi]}{1+\iota}
\end{gathered}
$$

\section{Bibliografía}

Ball, L. (1999), "Policy rules for open economies", Monetary Policy Rules, J. Taylor (ed.), Chicago, University of Chicago Press.

Barhoumi, K. (2006), "Differences in long run exchange rate pass-through into import prices in developing countries: An empirical investigation", Economic Modelling, vol. 23, $\mathrm{N}^{\circ} 6$, Amsterdam, Elsevier.

Batini, N., R. Harrison y S.P. Millard (2001), "Monetary policy rules for an open economy", Norges Bank Working Paper, $N^{\circ} 2001 / 4$, Oslo, Norges Bank.

Byrne, J., A. Chavali y A. Kontonikas (2010), "Exchange rate pass through to import prices: panel evidence from emerging market economies", Business School - Economics Working Papers, $\mathrm{N}^{\circ}$ 2010/19, Glasgow, Universidad de Glasgow, junio.

Calvo, G.A. (1983), "Staggered prices in a utility-maximizing framework", Journal of Monetary Economics, vol. 12, $\mathrm{N}^{\circ} 3$, Amsterdam, Elsevier.

Caballero, R.J. y A. Krishnamurthy (2005), "Inflation targeting and sudden stops", The Inflation-Targeting Debate, B.S. Bernanke y M. Woodford (eds.), Chicago, University of Chicago Press.

Céspedes, L.F. y C. Soto (2005), "Credibility and inflation targeting in Chile", documento preparado para la Novena Conferencia Anual del Banco Central de Chile "Política monetaria bajo metas de inflación", Santiago de Chile, 20 y 21 de octubre.

Céspedes, L.F., R. Chang y A. Velasco (2004), "Balance sheets and exchange rate policy", American Economic Review, vol. 94, $\mathrm{N}^{\circ} 4$, Nashville, Tennessee, American Economic Association. (2003), "IS-LM-BP in the Pampas", IMF Staff Papers, vol. 50, Washington, D.C., Fondo Monetario Internacional (FMI).

Choundri, E.U. y D.S. Hakura (2006), "Exchange rate pass-through to domestic prices: Does the inflationary environment matter?, Journal of International Money and Finance, vol. 25, $\mathrm{N}^{\circ} 4$, Amsterdam, Elsevier.

Coricelli, F., B. Jazbec e I. Masten (2004), "Exchange rate pass-through in acceding countries: The role of exchange rate regimes", EUI Working Paper, N $^{\circ}$ 2004/16, Instituto Universitario Europeo.

Edwards, S. (2006), "The relationship between exchange rates and inflation targeting revisited", NBER Working Paper, $\mathrm{N}^{\circ} 12163$, Cambridge, Massachusetts, National Bureau of Economic Research (NBER), abril.

FMI (Fondo Monetario Internacional) (2013), Perspectivas de la economía mundial: Esperanzas, realidades, riesgos, Washington, D.C

Fraga, A., I. Goldfajn y A. Minella (2003), "Inflation targeting in emerging market economies", NBER Working Paper, N ${ }^{\circ} 10019$, Cambridge, Massachusetts, National Bureau of Economic Research.

García-Solanes, J. y F. Torrejón-Flores (2012), "La fijación de metas de inflación da buenos resultados en América Latina", Revista de la CEPAL, $\mathrm{N}^{\circ} 106$ (LC/G.2518-P), Santiago de Chile.
Korhonen, I. y P. Wachtel (2006), "A note on exchange rate passthrough in cIs countries", Research in International Business and Finance, vol. 20, $\mathrm{N}^{\circ} 2$, Amsterdam, Elsevier.

Mihaljek, D. y M. Klau (2008), "Exchange rate pass-through in emerging market economies: "What has changed and why?", BIS Papers, $\mathrm{N}^{\circ} 35$, Banco de Pagos Internacionales.

Mishkin, F.S. (2008), "Exchange rate pass-through and monetary policy", NBER Working Paper, $\mathrm{N}^{\circ}$ 13889, Cambridge, Massachusetts, National Bureau of Economic Research.

Mishkin, F.S. y M. Savastano (2002), "Monetary policy strategies for Latin America", Journal of Development Economics, vol. 66, $\mathrm{N}^{\circ}$ 2, Amsterdam, Elsevier.

Nogueira, R. (2007), "Inflation targeting and exchange rate passthrough", Economía Aplicada, vol. 11, $\mathrm{N}^{\circ}$ 2, São Paulo, Universidad de São Paulo.

Nogueira, R. y M. León-Ledesma (2011), "Does exchange rate passthrough respond to measures of macroeconomic instability?", Journal of Applied Economics, vol. 14, $\mathrm{N}^{\circ}$ 1, Amsterdam, Elsevier.

Obstfeld, M. y K. Rogoff (1995), "The mirage of fixed exchange rates", Journal of Economic Perspectives, vol. 9, $\mathrm{N}^{\circ} 4$, Nashville, Tennessee, American Economic Association.

Sek, S.K. y Z. Kapsalyamova (2008), "Exchange rate passthrough and volatility: Impacts on domestic prices in four Asian countries", MPRA Working Paper, $\mathrm{N}^{\circ} 11130$, Munich, University Library of Munich, octubre.

Svensson, L. (2000), "Open-economy inflation targeting", NBER Working Paper, $\mathrm{N}^{\circ}$ 6545, Cambridge, Massachusetts, National Bureau of Economic Research.

Takatoshi, I. y S. Kiyotaka (2008), "Exchange rate changes and inflation in post-crisis Asian countries: Vector Autorregression Analysis of the exchange rate pass-through", Journal of Money, Credit and Banking, vol. 40, $\mathrm{N}^{\circ} 7$, Wiley.

Taylor, J. (2000), "Low inflation pass-through and the pricing power of firms", European Economic Review, vol. 44, $\mathrm{N}^{\circ} 4$, Amsterdam, Elsevier.

Tovar, C.E. (2005), "The mechanics of devaluations and the output response in a DSGE model: How relevant is the balance sheet effect?", BIS Working Papers, N ${ }^{\circ}$ 192, Basilea, Banco de Pagos Internacionales.

Vega, M. y D. Winkelried (2005), "Inflation targeting and inflation behavior: A successful story", International Journal of Central Banking, vol. 1, $\mathrm{N}^{\circ} 3$.

Wollmershäuser, T. (2003), "Should central banks react to exchange rate movements? An analysis of the robustness of simple policy rules under exchange rate uncertainty", documento presentado en el II Workshop on Macroeconomic Policy Research, Budapest, 2 y 3 de octubre.

Woodford, M. (2003), Interest and Prices: Foundations of a Theory of Monetary Policy, Princeton, Princeton University Press. 